\title{
STANDPUNKT
}

\section{Fragwürdige Instrumentalisierung von Menschenhandel}

\author{
Juanita Henning
}

W /enn etwas in Deutschland Konjunktur hat, dann die parteiübergreifende gesellschaftliche Dramatisierung von Menschenhandel. Allerdings: Das krasse Missverhältnis zwischen den angeblich 120.000 bis 200.000 jährlich nach Deutschland verschleppten Opfern und den lediglich rund 900 Menschenhandels-Opfern der hiesigen Kriminalstatistik verweist auf eine Glaubwürdigkeitslücke, die auch durch permanenten Hinweis auf die Dunkelzifferproblematik nicht kaschiert werden kann.

Die großzügig mit Schätzungen und Vermutungen operierende öffentliche Debatte zum Menschenhandel beißt sich mit der schnöden Empirie der Einwilligung und Freiwilligkeit hierher migrierender Menschen. Erst jüngst hat Annette Herz vom Freiburger Max Planck Institut konstatiert, dass die als »Menschenhändler « titulierten Personen von den Betroffenen (von »Opfern« spricht sie nicht!) als »willkommene Unterstützer bei Migration, Arbeitssuche, Unterbringung und/oder Prostitutionsaufnahme« angesehen würden.

Von Menschenhandel, mit dem man eigentlich gegen den Willen der Betroffenen ausgeübte Gewalt assoziiert, bleibt wenig übrig. Selbst das BKA räumt ein, dass ein Viertel bis ein Drittel der offiziell als »Opfer « bezeichneten Personen freiwillig hier ist. Und nicht wenigen von der Polizei aufgegriffenen, in der Prostitution tätigen Frauen mag es vorteilhaft erscheinen, sich als »Opfer« zu präsentieren, zumal Prostituierte nur in dieser Rolle gesellschaftlich Anerkennung erfahren.

Ich arbeite seit 14 Jahren in Frankfurt/Main mit Prostitutionsmigrantinnen. Diejenigen, die ich in dieser Zeit nach gängiger Sichtweise als "gehandelte Opfer « hätte bezeichnen müssen, kann ich an den Fingern einer Hand abzählen. Sie waren dabei keineswegs Opfer eines prostitutionsspezifischen Menschenhandels, sondern durch spezifische Umstände der Migration gewaltsam in ihren Persönlichkeitsrechten beeinträchtigt. Dies zu ahnden hätte es keiner besonderen Strafbestimmungen zum Menschenhandel bedurft.

Die hiesige Gesetzgebung zum Menschenhandel - in alter wie in neuer Fassung - zielt im Kern gegen eine auf Freiwilligkeit basierende Migration. Anwendung von Zwang und Gewalt ist keine notwendige Voraussetzung für die Erfüllung des Straftatbestands. Das Bringen in Prostitution bzw. unterbezahlte Beschäftigung unter Ausnutzung einer »Zwangslage« oder »auslandspezifischer Hilflosigkeit« reicht aus. Die Kriterien dafür sind niedrigschwellig definiert. Und: »Die Zwangslage muss nicht objektiv bestehen, wohl aber von den Opfern subjektiv empfunden werden.« (Tröndle/Fischer) Solche Deutungen verkehren den behaupteten Opferschutz in eine Kriminalisierung der Vermittler. "Auslandsspezifische Hilflosigkeit « liegt schon vor bei mangelnden Sprachkenntnissen oder wenn Betroffene bei Unterkunft und Verpfle- gung auf die »Täter « angewiesen sind. Nach solcher Logik könnten sämtliche »Gast«arbeiter der 50er Jahre als Menschenhandelsopfer und der damalige deutsche Staat als Verbrechersyndikat bezeichnet werden.

Die 2005 in Kraft getretenen neuen Strafbestimmungen zum Menschenhandel übersetzen in nationales Recht, was das UN-Menschenhandelsprotokoll in Art. 3a und der EU-Rahmenbeschluss von 2002 in Art. 1 Abs. 2 offen aussprechen: »Das Einverständnis eines Opfers von Menschenhandel zur beabsichtigten oder tatsächlich vorliegenden Ausbeutung ist unerheb-

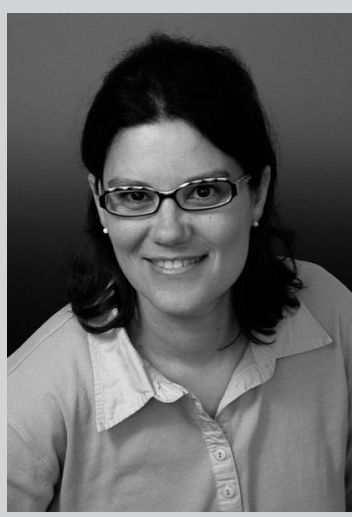

Juanita Henning, Dipl. Sozialarbeiterin und Mitarbeiterin des Vereins Doña Carmen e.V. lich«, wenn eine Anwerbung unter "Missbrauch einer Machtstellung oder Ausnutzung einer Position der Schwäche« erfolgt. Wenn aber Zwang und Gewalt einerseits, »Machtmissbrauch « und »Ausnutzung besonderer Hilflosigkeit « andererseits gleichermaßen konstitutiv für Menschenhandel sind, wird die Unterscheidung zwischen "Zwang « und »Freiwilligkeit« systematisch eingeebnet. Was bleibt, ist der Zwang und der Vorwurf des »Menschenhandels«, um freiwillige Migration zu kriminalisieren. Welche Migrationsbewegung in der Geschichte war jemals frei von der »Ausnutzung einer Position der Schwäche«? Hätte man sie deshalb strafrechtlich bekämpfen sollen?

»Menschenhandel« verkommt zu einem vom ursprünglichen Wortsinn losgelösten, für vielfältige politische Instrumentalisierung nützlichen Plastikbegriff. Über die Menschenhandels-Paragrafen ist auch das bundesdeutsche Strafrecht instrumentalisiert. Geht es doch darum, »die Sex- und Arbeitsmärkte einer deutlich sichtbaren Überwachung zu unterstellen « und die »Nachfrage nach sexuellen Dienstleistungen« zu verringern (»Brüsseler Erklärung« der EU von 2002). Frauenberatungsstellen, die mit der Polizei kooperieren und sich an Bordellrazzien beteiligen, sollen künftig statt »Opferzeuginnen« gewinnen »internationale Informantennetze aufbauen«.

Neben der Kontrolle von Arbeitsmärkten und Migration sind AntiMenschenhandels-Kampagnen immer schon gegen Prostitution an sich gerichtet. Die Figur des Menschenhändlers als globalisierte Version des Zuhälters: Wen wundert's, dass unter solchen Bedingungen das Prostitutionsgesetz der rotgrünen Bundesregierung zu einem Flop wurde? 